\title{
Angioarchitectures and Hemodynamic Characteristics of Posterior Communicating Artery Aneurysms and Their Association with Rupture Status
}

\author{
(D)B.J. Chung, (D) R. Doddasomayajula, (D)F. Mut, (D)F. Detmer, (D) M.B. Pritz, (D)F. Hamzei-Sichani, (D)W. Brinjikji, (DD.F. Kallmes, \\ (D)C.M. Jimenez, (D).M. Putman, and (D).R. Cebral
}

\begin{abstract}
BACKGROUND AND PURPOSE: Intracranial aneurysms originating at the posterior communicating artery are known to have high rupture risk compared with other locations. We tested the hypothesis that different angioarchitectures (ie, branch point configuration) of posterior communicating artery aneurysms are associated with aneurysm hemodynamics, which in turn predisposes aneurysms to rupture.

MATERIALS AND METHODS: A total of 313 posterior communicating artery aneurysms (145 ruptured, 168 unruptured) were studied with image-based computational fluid dynamics. Aneurysms were classified into different angioarchitecture types depending on the location of the aneurysm with respect to parent artery bifurcation. Hemodynamic characteristics were compared between ruptured and unruptured aneurysms, as well as among aneurysms with different angioarchitectures.
\end{abstract}

RESULTS: Angioarchitecture was associated with rupture $(P=.003)$. Ruptured aneurysms had higher, more concentrated, and more oscillatory wall shear stress distributions (maximum wall shear stress, $P<.001$; shear concentration index, $P<.001$; mean oscillatory shear index, $P<.001$ ), stronger and more concentrated inflow jets (represented as $Q, P=.01$; inflow concentration index, $P<.001$ ), and more complex and unstable flow patterns (vortex core length, $P<.001$; proper orthogonal decomposition entropy, $P<.001$ ) compared with unruptured aneurysms. These adverse conditions were more common in aneurysms with bifurcation-type angioarchitectures compared with those with lateral or sidewall angioarchitectures. Interestingly, ruptured aneurysms also had lower normalized mean wall shear stress $(P=.02)$ and minimum wall shear stress $(P=.002)$ than unruptured aneurysms.

CONCLUSIONS: High-flow intrasaccular hemodynamic characteristics, commonly found in bifurcation-type angioarchitectures, are associated with the posterior communicating artery aneurysm rupture status. These characteristics include strong and concentrated inflow jets, concentrated regions of elevated wall shear stress, oscillatory wall shear stress, lower normalized wall shear stress, and complex and unstable flow patterns.

ABBREVIATIONS: PCOM = posterior communicating artery; WSS = wall shear stress

O $\mathrm{n}$ average, the combined risk carried by aneurysm treatment far exceeds the annual risk of aneurysm rupture. ${ }^{1-3}$ As such, the best clinical practice would be to treat only those aneurysms

Received March 15, 2017; accepted after revision June 9.

From the Bioengineering Department (B.J.C., R.D., F.M., F.D., M.B.P., J.R.C.), George Mason University, Fairfax, Virginia; Department of Neurosurgery (F.H.-S.), Mt. Sinai Medical Center, New York, New York; Department of Radiology (W.B., D.F.K.), Mayo Clinic, Rochester, Minnesota; Neurosurgery Department (C.M.J.), University of Antioquia, Medellín, Colombia; and Interventional Neuroradiology Unit (C.M.P.) Inova Fairfax Hospital, Falls Church, Virginia.

This work was supported by the National Institutes of Health/National Institute of Neurological Disorders and Stroke (NIH-NINDS) grant \#R21NS094780.

Please address correspondence to Juan R. Cebral, PhD, Bioengineering Department, Volgenau School of Engineering, George Mason University, 4400 University Dr, MSN 2A1, Fairfax, VA 22030; e-mail: jcebral@gmu.edu

- Indicates open access to non-subscribers at www.ajnr.org

Indicates article with supplemental on-line photo.

http://dx.doi.org/10.3174/ajnr.A5358 that are relatively likely to rupture. Today, aneurysm size and location drive most clinical decisions, with consideration also given to surgical accessibility/endovascular feasibility, prior history of subarachnoid hemorrhage, family history, and patient age and general health. ${ }^{4}$ However, small aneurysms clearly are not entirely benign because the mean size of ruptured aneurysms is far smaller than $10 \mathrm{~mm}$, and many are $4 \mathrm{~mm}$ or less in diameter. ${ }^{5}$ Understanding the mechanisms that degrade the aneurysm wall and induce progression and eventual rupture or, alternatively, successful remodeling and repair that stabilizes the aneurysm, carries the promise of improving aneurysm evaluation as well as the development of novel therapies that target specific pathways of these mechanisms.

The posterior communicating artery (PCOM) is a common site for aneurysm development. PCOM aneurysms account for approximately $25 \%$ of all intracranial aneurysms. ${ }^{6}$ Aneurysms at 


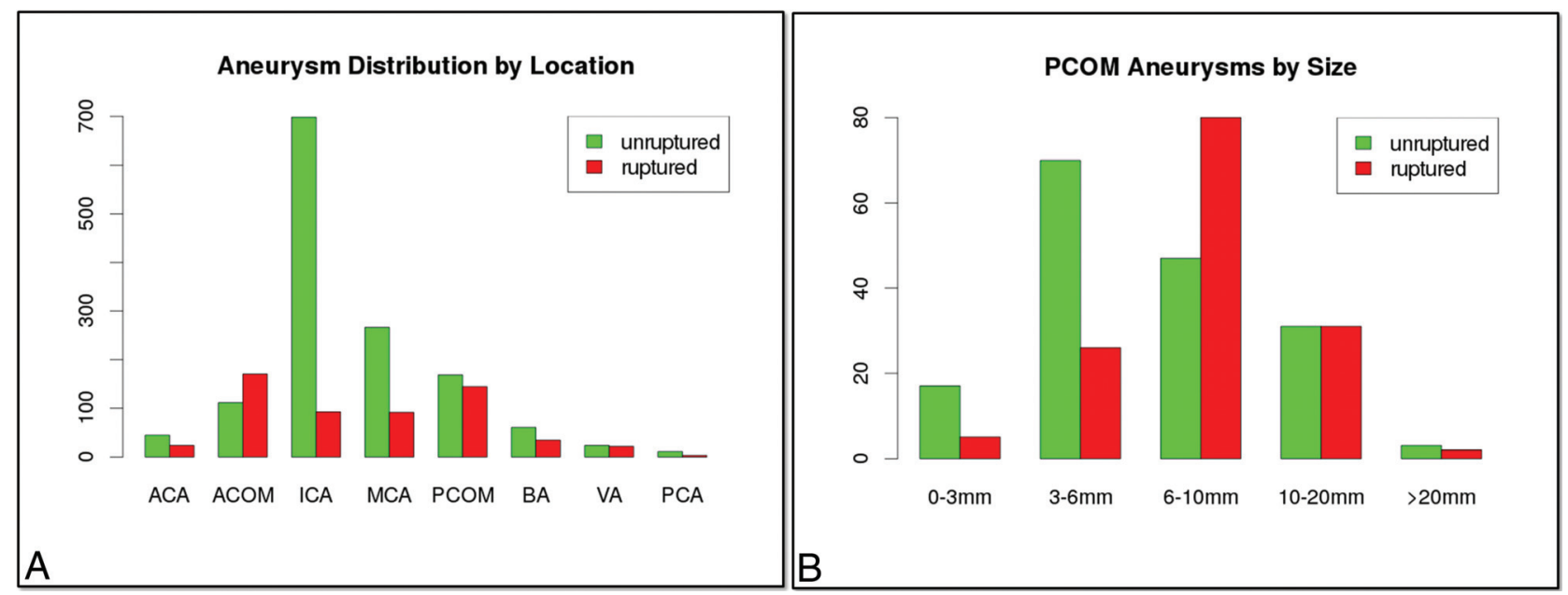

FIG 1. A, Distribution of all ruptured and unruptured aneurysms in our data base by aneurysm location, and $B$, distribution of ruptured and unruptured PCOM aneurysms by size. ACA indicates anterior cerebral artery; ACOM, anterior communicating artery; BA, basilar artery; PCA, posterior cerebral artery; VA, vertebral artery.

this location have larger rupture risk than aneurysms at other locations such as the MCA or other segments of the ICA. ${ }^{7,8}$ As such, clinicians often need to decide whether to treat PCOM aneurysms, but reliable aneurysm-specific parameters to guide and support decision making are lacking. A previous study showed that PCOM aneurysms, along with anterior communicating artery and basilar tip aneurysms, occur more frequently in patients with an anatomic variant or incomplete circle of Willis. ${ }^{9}$ A recent study found that inflow angle and the aneurysm area under low wall shear stress (WSS) were independently associated with rupture. ${ }^{10}$ These studies support the idea that hemodynamics are largely influenced by the local geometry of the vessels and aneurysm configurations and that they may play an important role in the development and evolution of intracranial aneurysms at this location.

To further test this hypothesis, the purpose of this study was to analyze the hemodynamic characteristics of ruptured and unruptured PCOM aneurysms and their relationship to the anatomic configuration of the aneurysm with respect to the ICA-PCOM bifurcation (ie, its angioarchitecture). The objective was to identify distinguishing characteristics that could be used to understand the underlying mechanisms of aneurysm evolution and, ultimately, use them to improve current risk assessment of PCOM aneurysms.

\section{MATERIALS AND METHODS \\ Data Base}

We have constructed a data base of intracranial aneurysms that have been imaged with $3 \mathrm{D}$ rotational angiography. In addition to the $3 \mathrm{D}$ rotational angiography images, the data base contains anonymized patient and aneurysm information such as aneurysm location, size, and rupture status as well as patient sex and age. Currently, the data base contains 2022 aneurysms in 1386 patients collected consecutively. All data have been anonymized, and the study has been approved by the George Mason institutional review board. The distribution of all ruptured and unruptured aneurysms in this data base by location is shown in Fig $1 \mathrm{~A}$. In this data base, PCOM aneurysms had the second largest rupture rate (46\%) after anterior communicating artery aneurysms (63\%) and were the third most common $(n=313)$ after ICA $(n=855)$ and MCA $(n=390)$ aneurysms. All PCOM aneurysms with known rupture status in this data base were selected for study. This selection resulted in a total of 313 PCOM aneurysms, 145 ruptured and 168 unruptured. The distribution of ruptured and unruptured PCOM aneurysms by size is presented in Fig $1 B$, showing that many of the ruptured aneurysms are small $(<6 \mathrm{~mm})$, and that even mediumsized aneurysms $(6-10 \mathrm{~mm})$ have large rupture rates $(63 \%)$.

\section{Angioarchitectures}

To test the hypothesis that different configurations of the ICA and PCOM arteries in relation to the location of the aneurysm can have important effects on intra-aneurysmal hemodynamics and, consequently, on aneurysm rupture, all PCOM aneurysms were classified into a set of angioarchitecture types presented in Fig 2. Aneurysms in type 3 were also classified into 2 subcategories: a) aneurysmal lesion, where the aneurysmal dilation occurs along an axis different from the PCOM axis, and b) infundibula, where the dilation is mainly aligned with the PCOM axis. The classification was done visually by inspection of volume-rendered 3 D rotational angiography images by 2 independent observers, and disagreements were resolved by consensus. To verify the invisibility of the PCOM in aneurysms of type 6, the transfer function of the volume rendering was interactively varied to enhance structures of low intensity. Examples of aneurysms in each angioarchitecture type are presented in the On-line Figure.

A posteriori, to simplify the analysis and increase the statistical power, aneurysms of types 2,5 , and 8 were combined into a "bifurcation" group, whereas types 1, 3a, 4, 6, and 7 were combined into a "lateral" group. In the lateral group, the aneurysm orifice is on the ICA (or the PCOM for type 1) and away from the bifurcation, whereas in the bifurcation group, the aneurysm ostium is near the bifurcation, hence their names. Infundibula (type 3b) were not considered for further analysis.

\section{Models}

Computational fluid dynamics models of all 313 PCOM aneurysms were created by using the patient-specific vascular ge- 


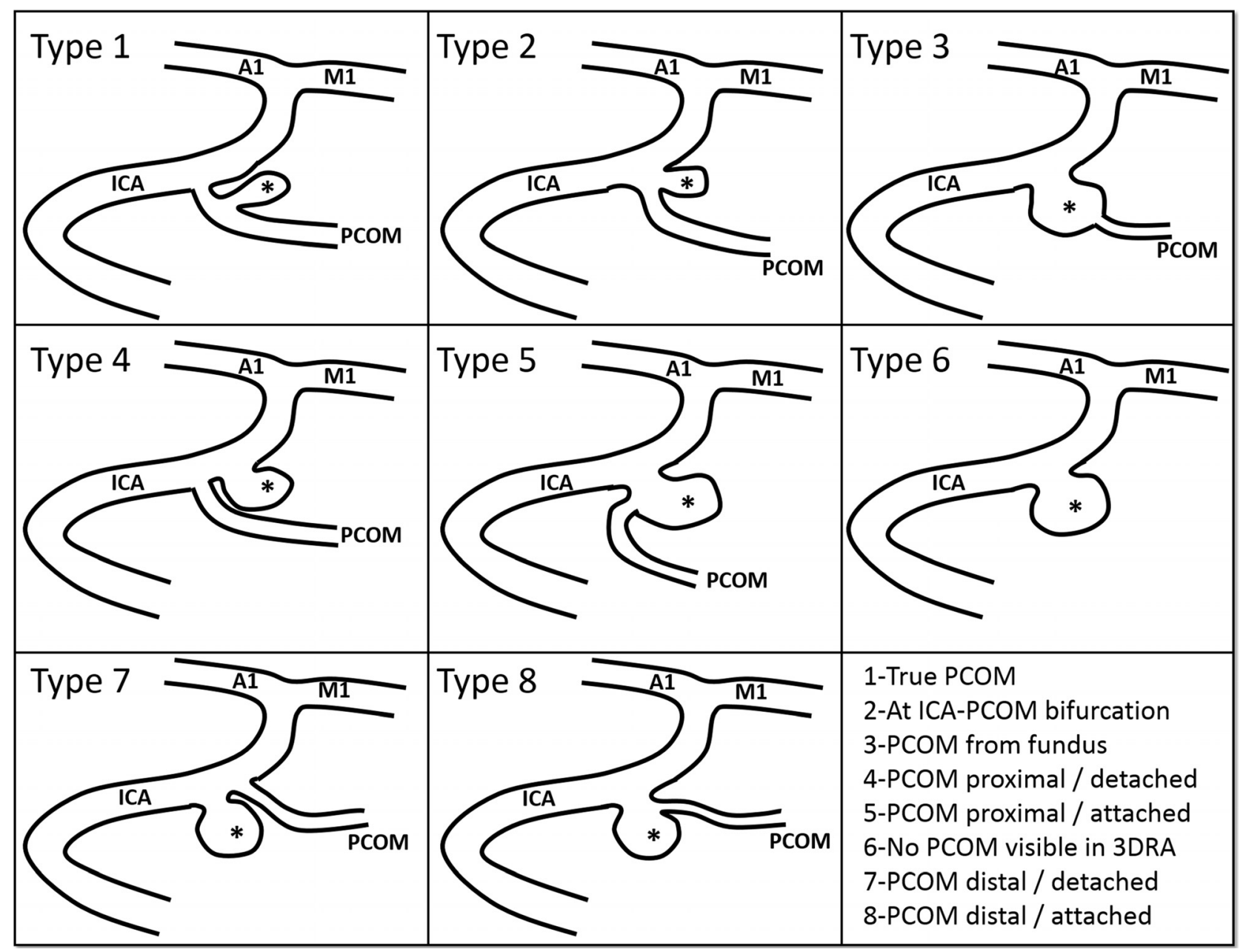

FIG 2. Angioarchitecture types of PCOM aneurysms. Al indicates proximal segment of anterior cerebral artery; M1, proximal segment of the MCA. The aneurysm is marked with an asterisk.

ometries reconstructed from the corresponding 3D rotational angiography images. ${ }^{11}$ The models were extended proximally to the cavernous segment of the ICA and distally to the M1 segment of the MCA and the Al segment of the anterior cerebral artery. It was verified that the reconstructed geometries adequately represented the assigned aneurysm angioarchitectural class. The geometries were meshed with tetrahedral elements with a minimum resolution of $200 \mu \mathrm{m}$, resulting in computational grids ranging from 2-5 million elements.

Numeric simulations based on the 3D incompressible NavierStokes equations were carried out assuming Newtonian viscosity and neglecting vessel wall compliance. Pulsatile inflow conditions were prescribed at the inlet boundary located at the proximal ICA. A flow waveform derived from phase-contrast MR measurements at the ICA of healthy subjects ${ }^{12}$ was scaled with the inlet crosssectional area. ${ }^{13}$ The Womersley velocity profile was used to prescribe Dirichlet boundary conditions. ${ }^{14}$ Outflow boundary conditions were selected to produce flow divisions consistent with Murray's principle of minimal work to avoid unrealistic jumps in the WSS from parent to daughter arterial branches. Simulations were run for 2 cardiac cycles with a time resolution of $10 \mathrm{~ms}$, and the results from the second cycle were used for analysis to discard transients associated with the initialization of the numeric calcu- lations. All simulations were carried out in parallel on shared memory computers by using an in-house fully implicit finite element solver. ${ }^{15}$

To quantitatively characterize the intrasaccular hemodynamic environments of the aneurysms and to compare ruptured and unruptured aneurysms, a set of flow variables (listed in Table 1) defined over the aneurysm region were computed. ${ }^{16,17}$

\section{Data Analysis}

The flow variables in Table 1 were used to test whether ruptured and unruptured PCOM aneurysms have different hemodynamic environments and whether different angioarchitectures are associated with different intra-aneurysmal flow characteristics. Twosided Wilcoxon rank sum (Mann-Whitney $U$ ) tests were used to compare the mean values of each continuous variable between the different aneurysm groups (ruptured/unruptured or different angioarchitectures). Differences were considered statistically significant if $P<.05$ (95\% confidence). The association between angioarchitecture categories and aneurysm rupture rate was tested by using contingency table analysis (Fisher test for $2 \times 2$ tables, $\chi^{2}$ test for $\mathrm{N} \times \mathrm{M}$ tables). Statistical analysis was carried out in $\mathrm{R}$ software (http://www.r-project.org/). ${ }^{18}$ 


\section{RESULTS}

\section{Angioarchitecture Associated with Rupture}

The numbers of ruptured and unruptured PCOM aneurysms of each angioarchitecture are presented in Fig $3 A$. It can be seen that the largest proportions of ruptured aneurysms are in types 2 and 5 (bifurcation aneurysms), whereas the largest proportion of unruptured aneurysms is in type 6, where the PCOM is not visible and the aneurysm can be considered a sidewall or lateral aneurysm. Aneurysms with angioarchitectures 1, 4, 7, and 8 were quite uncommon. Angioarchitecture was associated with aneurysm rupture according to contingency table analysis using the $\chi^{2}$ test $(P=.003)$.

Table 1: Flow variables used to characterize different aspects of the hemodynamic environment of ruptured and unruptured PCOM aneurysms ${ }^{a}$

\begin{tabular}{lccc}
\hline \multicolumn{1}{c}{ Variable } & Unruptured & Ruptured & $P$ \\
\hline Wall shear stress distribution characteristics & & & \\
WSSmin, dyne $/ \mathrm{cm}^{2}$ & $0.64 \pm 1.39$ & $0.29 \pm 0.74$ & $.002^{\mathrm{b}}$ \\
WSSmean, dyne $/ \mathrm{cm}^{2}$ & $22.34 \pm 20.16$ & $24.17 \pm 23.21$ & .59 \\
WSSnorm & $0.51 \pm 0.33$ & $0.43 \pm 0.28$ & $.02^{\mathrm{b}}$ \\
WSSmax, dyne/cm ${ }^{2}$ & $231.54 \pm 172.1$ & $341.30 \pm 299.9$ & $<.001^{\mathrm{b}}$ \\
MWSSnorm & $5.71 \pm 5.05$ & $6.10 \pm 3.18$ & $<.001^{\mathrm{b}}$ \\
OSImax & $0.261 \pm 0.137$ & $0.336 \pm 0.102$ & $<.001^{\mathrm{b}}$ \\
OSImean & $0.013 \pm 0.014$ & $0.017 \pm 0.015$ & $<.001^{\mathrm{b}}$ \\
SCI & $5.76 \pm 8.50$ & $7.04 \pm 7.17$ & $<.001^{\mathrm{b}}$ \\
LSA & $52.32 \pm 31.91$ & $59.63 \pm 30.46$ & .05 \\
Aneurysm inflow characteristics & & & \\
Q, cm ${ }^{3} / \mathrm{s}$ & $0.71 \pm 0.85$ & $0.88 \pm 0.99$ & $.01^{\mathrm{b}}$ \\
ICI & $0.649 \pm 0.785$ & $0.782 \pm 0.674$ & $.02^{\mathrm{b}}$ \\
Intra-aneurysmal flow characteristics & & & \\
Vmax, cm/s & $67.89 \pm 33.84$ & $95.73 \pm 59.21$ & $<.001^{\mathrm{b}}$ \\
VE, cm/s & $9.75 \pm 7.39$ & $10.71 \pm 7.67$ & .18 \\
VO, $1 / \mathrm{s}$ & $320.47 \pm 274.3$ & $323.66 \pm 254.7$ & .57 \\
SR, $1 / \mathrm{s}$ & $236.26 \pm 201.9$ & $235.06 \pm 182.4$ & .67 \\
CORELEN, cm & $1.837 \pm 2.484$ & $3.133 \pm 2.982$ & $<.001^{\mathrm{b}}$ \\
PODENT & $0.179 \pm 0.140$ & $0.206 \pm 0.117$ & $<.001^{\mathrm{b}}$ \\
\hline
\end{tabular}

Note:-CORELEN indicates vortex core length - flow complexity; ICI, inflow concentration index; LSA, low shear area (\% of aneurysm sac); MWSSnorm, maximum normalized WSS; OSImax, maximum oscillatory shear index; OSImean, mean oscillatory shear index; PODENT, proper orthogonal decomposition entropy-flow stability; $\mathrm{Q}$, aneurysm inflow rate; $\mathrm{SCI}$, shear concentration index; SR, mean aneurysm shear rate; VE, mean aneurysm velocity; Vmax, maximum aneurysm velocity; VO, mean aneurysm vorticity; WSSmax, maximum WSS; WSSmean, average WSS; WSSmin, minimum WSS; WSSnorm, normalized WSS.

${ }^{a}$ Values given are mean \pm standard deviation, and $P$ values correspond to the Mann-Whitney $U$ test.

${ }^{\mathrm{b}}$ Statistically significant association.
The distributions of ruptured and unruptured aneurysms in the combined bifurcation and lateral angioarchitecture groups are presented in Fig $3 B$. Fig $3 B$ shows that the bifurcation group has a larger rupture rate than the lateral group (54.6\% versus $36.4 \%)$. This association is statistically significant according to the Fisher exact test for the $2 \times 2$ contingency table $(P=.004)$.

\section{Hemodynamic Characteristics Associated with Rupture}

Results of the association between hemodynamic variables and aneurysm rupture are presented in Table 1. Irrespective of angioarchitecture, compared with unruptured aneurysms, ruptured PCOM aneurysms had larger maximum WSS $(P<.001$; normalized maximum WSS, $P<.001)$, more concentrated WSS distributions (shear concentration index, $P<.001$ ), more oscillatory WSS (maximum oscillatory shear index, $P<.001$; mean oscillatory shear index, $P<.001$ ), larger inflow rates (represented as $\mathrm{Q}, P=.01$ ) and maximum velocity $(P<.001)$, more concentrated inflow jets (inflow concentration index, $P<.001)$, more complex flows (vortex core length, $P<.001$ ), and more unstable flow patterns (proper orthogonal decomposition entropy, $P<.001)$. Interestingly, ruptured aneurysms also had lower normalized mean WSS $(P=.02)$ and minimum WSS $(P=.002)$ than unruptured aneurysms.

\section{Hemodynamic Characteristics Associated with Angioarchitectures} Results of the association between hemodynamic variables and rupture of aneurysms of different angioarchitectures are summarized in Table 2. Table 2 indicates which hemodynamic variables were
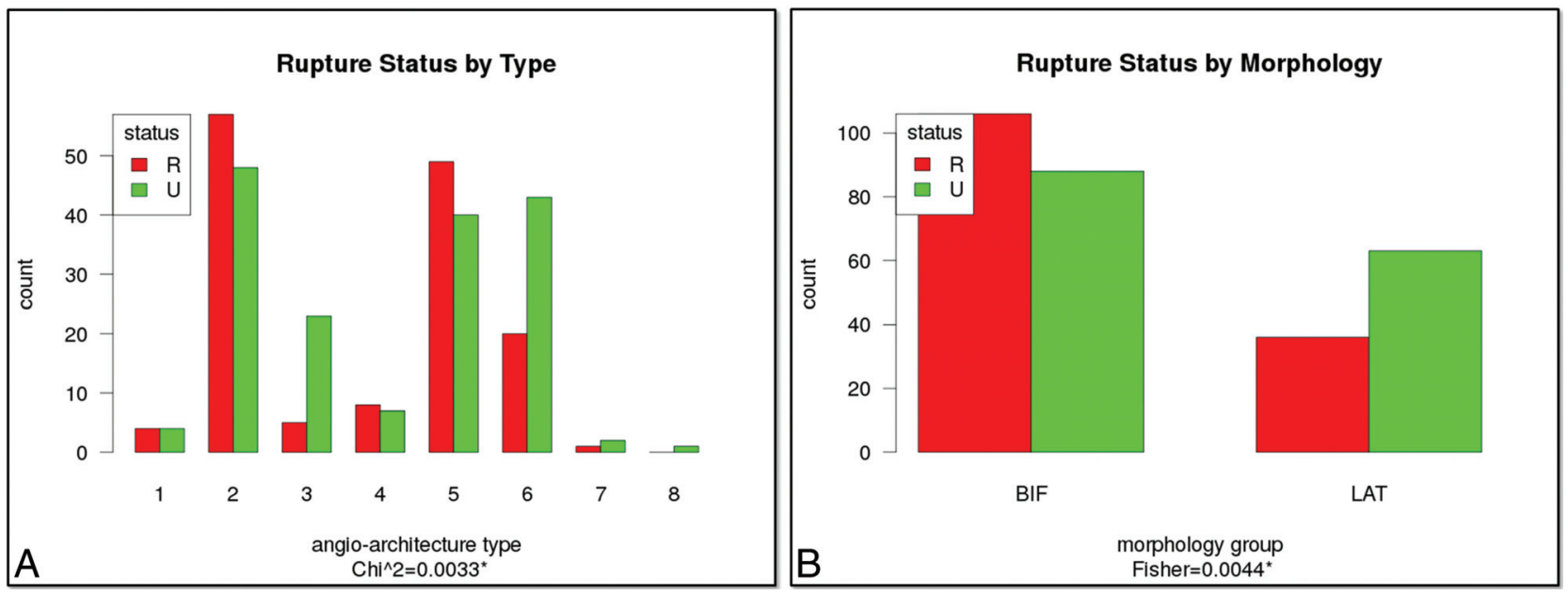

FIG 3. $A$, Number of ruptured and unruptured PCOM aneurysms in each angioarchitecture type, and $B$, number of ruptured and unruptured PCOM aneurysms in "bifurcation" (types 2, 5, and 8 combined) and "lateral" (types 1, 3, 4, 6, and 7 combined) angioarchitectures. BIF indicates bifurcation; LAT, lateral. 
Table 2: Summary of univariate statistical tests comparing the means between ruptured and unruptured aneurysms considering all aneurysms, subdividing by angioarchitecture types 1-8, and combining angioarchitectures into "bifurcation" and "lateral" configurations $^{\mathrm{a}}$

\begin{tabular}{|c|c|c|c|c|c|c|c|c|c|c|c|c|}
\hline \multirow[b]{2}{*}{ Variable } & \multirow[b]{2}{*}{ All } & \multicolumn{8}{|c|}{ Angioarchitecture Type } & \multicolumn{3}{|c|}{$\begin{array}{c}\text { Combined } \\
\text { Angioarchitectures }\end{array}$} \\
\hline & & 1 & 2 & 3 & 4 & 5 & 6 & 7 & 8 & BIF & LAT & BIF vs LAT \\
\hline $\begin{array}{l}\text { WSSmin } \\
\text { WSSmean }\end{array}$ & $U$ & & & & & & $U$ & & & & & $\mathrm{~L}$ \\
\hline WSSnorm & $U$ & & & & & & & & & & $U$ & \\
\hline WSSmax & $\mathrm{R}$ & & $\mathrm{R}$ & & & & $\mathrm{R}$ & & & $\mathrm{R}$ & $\mathrm{R}$ & \\
\hline MWSSnorm & $\mathrm{R}$ & & & & & & & & & $\mathrm{R}$ & & B \\
\hline OSImax & $\mathrm{R}$ & & & & & & $\mathrm{R}$ & & & $\mathrm{R}$ & $\mathrm{R}$ & B \\
\hline OSImean & $\mathrm{R}$ & & & & & & $\mathrm{R}$ & & & & $\mathrm{R}$ & B \\
\hline $\mathrm{SCl}$ & $\mathrm{R}$ & & & & & & $\mathrm{R}$ & & & & & B \\
\hline \multicolumn{13}{|l|}{ LSA } \\
\hline$Q$ & $\mathrm{R}$ & & & & & & & & & & & B \\
\hline $\mathrm{ICl}$ & $\mathrm{R}$ & & & & & & & & & $\mathrm{R}$ & & B \\
\hline Vmax & $\mathrm{R}$ & & $\mathrm{R}$ & & & $\mathrm{R}$ & $\mathrm{R}$ & & & $\mathrm{R}$ & $\mathrm{R}$ & \\
\hline \\
\hline \multicolumn{13}{|l|}{ VO } \\
\hline \multicolumn{13}{|l|}{ SR } \\
\hline CORELEN & $\mathrm{R}$ & & $\mathrm{R}$ & & & $\mathrm{R}$ & $\mathrm{R}$ & & & $\mathrm{R}$ & $\mathrm{R}$ & B \\
\hline PODENT & $\mathrm{R}$ & & & & & & $\mathrm{R}$ & & & & & B \\
\hline
\end{tabular}

Note:-BIF indicates bifurcation; CORELEN, vortex core length - flow complexity; ICI, inflow concentration index; LAT, lateral; LSA, low shear area (\% of aneurysm sac); MWSSnorm, maximum normalized WSS; OSImax, maximum oscillatory shear index; OSImean, mean oscillatory shear index; PODENT, proper orthogonal decomposition entropy — flow stability; $\mathrm{Q}$, aneurysm inflow rate; SCl, shear concentration index; SR, mean aneurysm shear rate; $V E$, mean aneurysm velocity; $V$ max, maximum aneurysm velocity VO, mean aneurysm vorticity; WSSmax, maximum WSS; WSSmean, average WSS; WSSmin, minimum WSS; WSSnorm, normalized WSS.

${ }^{a} \mathrm{R}$ indicates mean value significantly larger $(P<0.05)$ in ruptured compared with unruptured aneurysms. $U$ indicates mean value significantly larger $(P<0.05)$ in unruptured compared with ruptured aneurysms. B indicates mean value significantly larger $(P<0.05)$ in BIF compared with LAT aneurysms. L indicates mean value significantly larger $(P<$ $0.05)$ in LAT compared with BIF aneurysms. Empty cells indicate no statistically significant difference.

significantly different between ruptured and unruptured aneurysms of each angioarchitecture type and whether the value was larger in the ruptured or unruptured group or between the combined bifurcation and lateral groups. In general, bifurcation aneurysms had larger maximum normalized WSS $(P<.001)$, lower minimum WSS $(P=.001)$, more oscillatory WSS $(P=$ .0033; mean oscillatory WSS, $P=.01$ ), more concentrated WSS (shear concentration index, $P<.001$ ), larger and more concentrated inflow jet $(\mathrm{Q}, P=.001$; inflow concentration index, $P<$ .001 ), and more complex and unstable flows (vortex core length, $P=.003$; proper orthogonal decomposition entropy, $P=.01$ ) than lateral aneurysms.

\section{DISCUSSION}

Aneurysm evolution is thought to be governed by the progressive degradation of the wall in response to abnormal hemodynamic and biomechanical stimuli. ${ }^{19}$ Previous studies of aneurysms in other locations have found angioarchitectural differences between ruptured and unruptured aneurysms. In basilar tip aneurysms, bifurcation angles between the basilar and posterior cerebral arteries and the diameters of the posterior cerebral arteries were different between ruptured and unruptured aneurysms. ${ }^{20}$ In aneurysms at the MCA bifurcation ${ }^{21}$ and the anterior communicating complex, ${ }^{22}$ neck location, flow deviation, and relation of neck/daughter artery were different between ruptured and unruptured aneurysms. Therefore, it is logical to ask whether different angioarchitectures could be associated with different hemodynamic conditions that in turn could predispose the aneurysm to rupture or stabilization.

Our current study found that ruptured PCOM aneurysms had distinct hemodynamic environments compared with unruptured aneurysms. Specifically, the hemodynamics in ruptured aneurysms were characterized by concentrated elevations of WSS, oscillatory WSS, stronger and more concentrated inflow jets, and more complex and unstable flow patterns than in unruptured aneurysms. Similar trends were observed when subdividing by angioarchitectural types, though in some types, the trends did not reach statistical significance, likely because of small sample sizes. This suggests that hemodynamic environments with these characteristics may be more harmful to the wall, thus increasing the likelihood of rupture. This reasoning is supported by previous studies that found similar hemodynamic characteristics associated with weakened aneurysm walls ${ }^{23}$ as well as inflammation and other histologic changes of the wall. ${ }^{24}$

Interestingly, ruptured aneurysms also had lower normalized WSS and minimum WSS than unruptured aneurysms. Therefore, our results are in agreement with previous studies that pointed to "high WSS" mechanisms as responsible for aneurysm rupture ${ }^{25}$ as well as with studies that proposed "low WSS" mechanisms. ${ }^{10,26,27}$ This finding suggests that previous results may not be conflicting or contradictory, but rather that the different metrics used in different studies characterize different aspects of the adverse flow environment. Both abnormally high and low WSS have been associated with processes that could degrade the aneurysm wall. ${ }^{28}$ Our study suggests that aneurysms with high local flow conditions (eg, high maximum WSS, high velocity, and complex and unstable flows) as well as low local flow conditions (eg, low normalized WSS and low minimum WSS) may be more likely to rupture.

Further, the current study showed that the proposed angioarchitecture types are associated with aneurysm rupture. In par- 

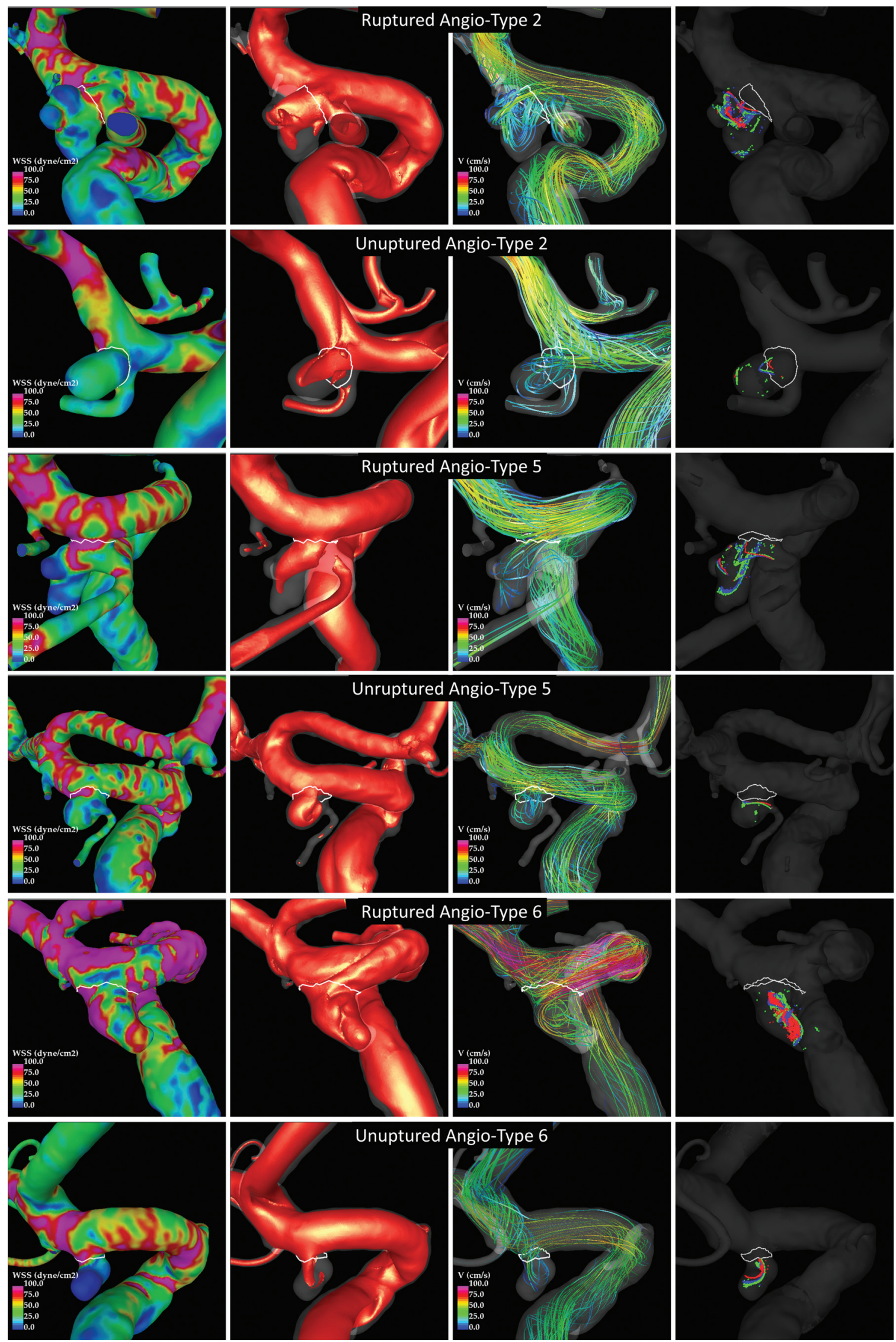

FIG 4. Examples of ruptured and unruptured aneurysms in the most common angioarchitecture classes (types 2,5 , and 6 ). Columns show, from left to right, respectively: WSS magnitude, inflow jet, flow pattern at peak systole, and vortex cores at 3 instants of the cardiac cycle. 
ticular, bifurcation-type aneurysms had significantly higher rupture rates than aneurysms that behave as lateral aneurysms. Furthermore, aneurysms in the bifurcation group had hemodynamic environments characterized by concentrated elevations of WSS, oscillatory WSS, larger and more concentrated inflow jets, and more complex and unstable flow patterns. This suggests that bifurcation angioarchitectures are more likely to generate these adverse intra-aneurysmal hemodynamic conditions that predispose the aneurysms to rupture.

It is conceivable that compared with more lateral or sidewall aneurysm configurations, bifurcation aneurysms are more likely to have stronger inflow jets directed toward the aneurysm fundus that create localized regions of concentrated WSS and spread into complex and unstable flow patterns. To illustrate this point, examples of ruptured and unruptured aneurysms of angioarchitecture types 2, 5, and 6 are presented in Fig 4.

Although the current sample was large enough to obtain statistically significant differences, some limitations in this study should be considered, and the results should be confirmed with data from other populations as well as with longitudinal data. The current study purposely focused only on PCOM aneurysms to eliminate confusion related to aneurysm location. However, the proposed angioarchitecture classification and corresponding hemodynamic analysis could be directly evaluated with aneurysms at other locations, such as the anterior choroidal artery, or extended to other locations, such as the MCA bifurcation or the superior cerebellar artery. The study was performed on a set from a data base of consecutive aneurysms that had undergone angiography and/or embolization over a 10 -year period. Selection bias related to referral patterns and indications for treatment may have led to the exclusion of important aneurysms subsets. In addition, a number of assumptions and approximations were made during the modeling process, including the approximations of the vascular geometry, the inflow conditions, the Newtonian rheology, and the rigid walls assumption.

\section{CONCLUSIONS}

High-flow intrasaccular hemodynamic characteristics are associated with PCOM aneurysm rupture. High-flow characteristics include strong and concentrated inflow jets, concentrated regions of elevated WSS, oscillatory WSS, and complex and unstable flow patterns. These adverse conditions are more commonly found in aneurysms with bifurcation-type angioarchitectures in contrast to aneurysms that have a hemodynamic behavior similar to sidewall aneurysms. Angioarchitecture and hemodynamic characteristics associated with rupture are useful to further understand the underlying mechanisms of aneurysm evolution and rupture and could potentially be used to refine the clinical evaluation of PCOM aneurysms and plan more specific and personalized therapeutic strategies.

Disclosures: Ravi Doddasomayajula—RELATED: Grants/Grants Pending: National Institutes of Health, Comments: research grant as mentioned in the manuscript*. Fernando Mut-RELATED: Grants/Grants Pending: National Institutes of Health*. Waleed Brinjikji-UNRELATED: Other: Marblehead Medical LLC, Comments: CEO of Marblehead Medical. David F. Kallmes-UNRELATED: Consultancy: Medtronic, Comments: steering committee participation*; Grants/Grants Pending: Microvention, Sequent Medical, NeuroSigma, Medtronic, Comments: preclinical research and clinical trials*. Christopher Putman-UNRELATED: Payment for Lectures (including service on Speakers Bureaus): Penumbra. Juan R. Cebral-RELATED: Grants/ Grants Pending: National Institutes of Health, Comments: research grant*; UNRELATED: Grants/Grants Pending: National Institutes of Health, Comments: research grants*. *Money paid to the institution.

\section{REFERENCES}

1. Kassell NF, Torner JC, Haley EC Jr, et al. The International Cooperative Study on the Timing of Aneurysm Surgery. Part 1: overall management results. J Neurosurg 1990;73:18-36 CrossRef Medline

2. Nishioka H, Torner JC, Graf CJ, et al. Cooperative study of intracranial aneurysms and subarachnoid hemorrhage: a long-term prognostic study. II. Ruptured intracranial aneurysms managed conservatively. Arch Neurol 1984;41:1142-46 CrossRef Medline

3. White PM, Wardlaw JM. Unruptured intracranial aneurysms. J Neuroradiol 2003;30:336-50 Medline

4. Greving JP, Wermer MJ, Brown RD Jr, et al. Development of the PHASES score for prediction of risk of rupture of intracranial aneurysms: a pooled analysis of six prospective cohort studies. Lancet Neurol 2014;13:59-66 CrossRef Medline

5. Weir B. Unruptured intracranial aneurysms: a review. J Neurosurg 2002;96:3-42 CrossRef Medline

6. Golshani K, Ferrell A, Zomorodi A, et al. A review of the management of posterior communicating artery aneurysms in the modern era. Surg Neurol Int 2010;1:88 CrossRef Medline

7. Cebral JR, Raschi M. Suggested connections between risk factors of intracranial aneurysms: a review. Ann Biomed Eng 2013;41:1366-83 CrossRef Medline

8. Weir B, Disney L, Karrison T. Sizes of ruptured and unruptured aneurysms in relation to their sites and the ages of patients. $\mathrm{J} \mathrm{Neu}$ rosurg 2002;96:64-70 CrossRef Medline

9. Songsaeng D, Geibprasert S, Willinsky R, et al. Impact of anatomical variations of the circle of Willis on the incidence of aneurysms and their recurrence rate following endovascular treatment. Clin Radiol 2010;65:895-901 CrossRef Medline

10. Lv N, Wang C, Karmonik C, et al. Morphological and hemodynamic discriminators for rupture status in posterior communicating artery aneurysms. PloS One 2016;11:e149906 CrossRef Medline

11. Cebral JR, Castro MA, Appanaboyina S, et al. Efficient pipeline for image-based patient-specific analysis of cerebral aneurysm hemodynamics: technique and sensitivity. IEEE Trans Med Imaging 2005;24:457-67 CrossRef Medline

12. Ford MD, Alperin N, Lee $\mathrm{SH}$, et al. Characterization of volumetric flow rate waveforms in the normal internal carotid and vertebral arteries. Physiol Meas 2005;26:477-88 CrossRef Medline

13. Cebral JR, Castro MA, Putman CM, et al. Flow-area relationship in internal carotid and vertebral arteries. Physiol Meas 2008;29:585-94 CrossRef Medline

14. Taylor CA, Hughes TJR, Zarins CK. Finite element modeling of blood flow in arteries. Comp Meth App Mech Eng 1998;158:155-96 CrossRef

15. Mut F, Aubry R, Löhner R, et al. Fast numerical solutions of patientspecific blood flows in 3D arterial systems. Int J Numer Method Biomed Eng 2010;26:73-85 CrossRef Medline

16. Mut F, Löhner R, Chien A, et al. Computational hemodynamics framework for the analysis of cerebral aneurysms. Int J Numer Method Biomed Eng 2011;27:822-39 CrossRef Medline

17. Byrne G, Mut F, Cebral JR. Quantifying the large-scale hemodynamics of intracranial aneurysms. AJNR Am J Neuroradiol 2014;35: 333-38 CrossRef Medline

18. R Development Core Team. R: A Language and Environment for Statistical Computing. Vienna, Austria: R Foundation for Statistical Computing; 2016

19. Sforza DM, Putman CM, Cebral JR. Hemodynamics of cerebral aneurysms. Annu Rev Fluid Mech 2009;41:91-107 CrossRef Medline

20. Ambekar S, Madhugiri V, Bollam P, et al. Morphological differences between ruptured and unruptured basilar bifurcation aneurysms. J Neurol Surg B Skull Base 2013;74:91-96 CrossRef Medline

21. Sadatomo T, Yuki K, Migita K, et al. Morphological differences between 
ruptured and unruptured cases in middle cerebral artery aneurysms. Neurosurgery 2008;62:602-09; discussion 602-09 CrossRef Medline

22. Sadatomo T, Yuki K, Migita K, et al. The characteristics of the anterior communicating artery aneurysm complex by three-dimensional digital subtraction angiography. Neurosurg Rev 2006;29: 201-07 CrossRef Medline

23. Cebral JR, Duan X, Chung BJ, et al. Wall mechanical properties and hemodynamics of unruptured intracranial aneurysms. AJNR Am J Neuroradiol 2015;36:1695-703 CrossRef Medline

24. Cebral J, Ollikainen E, Chung BJ, et al. Flow conditions in the intracranial aneurysm lumen are associated with inflammation and degenerative changes of the aneurysm wall. AJNR Am J Neuroradiol 2017;38:119-26 CrossRef Medline

25. Cebral JR, Mut F, Weir J, et al. Quantitative characterization of the hemodynamic environment in ruptured and unruptured brain aneurysms. AJNR Am J Neuroradiol 2011;32:145-51 CrossRef Medline

26. Xiang J, Natarajan SK, Tremmel M, et al. Hemodynamic-morphologic discriminants for intracranial aneurysm rupture. Stroke 2011; 42:144-52 CrossRef Medline

27. Zhang Y, Jing L, Liu J, et al. Clinical, morphological, and hemodynamic independent characteristic factors for rupture of posterior communicating artery aneurysms. J Neurointerv Surg 2016;8:808-12 CrossRef Medline

28. Meng H, Tutino VM, Xiang J, et al. High WSS or low WSS? Complex interactions of hemodynamics with intracranial aneurysm initiation, growth, and rupture: toward a unifying hypothesis. AJNR Am J Neuroradiol 2014;35:1254-62 CrossRef Medline 